\title{
REVUE
}

\section{ORIENTATION DES RECHERCHES SUR LE LAIT EN FRANCE (1)}

\author{
par \\ G. MOCQUOT
}

Station Centrale de Mierobiologie et de Recherches Laitières

Le lait occupe en France, l'une des premières places parmi les différentes productions agricoles du pays. Son rôle dans la nutrition de l'enfant et de l'adulte - sous forme de lait en nature, de beurre ou de fromage - justifie l'intérêt qui s'attache aux moyens mis en œuvre pour le récolter, le conserver ou le transformer. D'autre part, le lait est un produit fragile et les divers procédés technologiques en usage, dont certains sont très anciens, ne peuvent être compris, améliorés et dans certains cas modifiés, qu'en faisant appel à'des notions intéressant plusieurs disciplines scientifiques parmi lesquelles on doit citer la bactériologie, la physiologie, la biochimie, la physique, l'hygiène.

Le but des études poursuivies par les laboratoires de la Recherche Agronomique est d'augmenter la somme de nos connaissances relatives au lait en ces divers domaines, tout en orientant les recherches dans le sens le plus favorable à la solution des problèmes pratiques. Ceux-ci étant à leur tour abordés plus spécialement sous l'angle particulier qui intéresse notre pays.

Les moyens consacrés aux recherches sur le lait dans le monde entier, depuis trois quart de siècle, ont été sans cesse en augmentant et ont déjà permis de rassembler une documentation assez considérable. En France, les travaux poursuivis par les premiers expérimentateurs : Pasteur, Duclaux, Lindet et plus tard Mazé, Porcher, Guittonneau ont apporté une contribution appréciable à nos connaissances.

Il suffit de rappeler l'origine du mot "pasteurisation ", qui désigne maintenant l'une des techniques essentielles de traitement du lait, pour comprendre le rôle primordial joú par les travaux des laboratoires dans la mise au point de procédés pratiques dont l'usage est aujourd'hui répandu partout.

Nous voudrions situer ici rapidement les divers problèmes que pose l'utilisation du lait et montrer ensuite dans quel sens on doit orienter les recherches pour permettre d'aboutir à des solutions, dont certaines peuvent être obtenues assez rapidement, alors que

(1) Annales Inst. Nat. Rech. Agro. (E. Technologie), 1952, p, 11. 
d'autres nécessitent des travaux de longue haleine, souvent indispensables pour arriver au but que l'on se propose.

\section{PROBLÈMES POSÉS PAR L'UTILISATION DU LAIT}

\section{Qualité bactériologique et teneur du lait et des produits laitiers en éléments nutritifs.}

Le problème de base, c'est de trouver les meilleures méthodes à suivre pour permettre que le lait, trait à la ferme chaque matin et chaque soir, puis rassemblé dans des laiteries d'importance variable et redistribué ensuite aux consommateurs sous forme de lait liquide ou d'autres produits, parvienne au centre de collecte avec une population bactérienne aussi réduite que possible. C'est le problème de l'asepsie à l'échelle industrielle. Ceci suppose, d'une part, que le matériel et les méthodes de travail mises en œuvre pour la collecte sont judicieusement choisis, d'autre part, qu'il existe des moyens pratiques de s'assurer à tous les stades que le résultat cherché — i. e. la bonne qualité du lait —, est effectivement atteint.

Cet aspect $d u$ problème laitier est absolument fondamental : par ailleurs, la dispersion en petites étables de la production française du lait, dispersion liée bien souvent à la structure même de nos différentes régions agricoles, rend la question plus difficile à résoudre que dans les pays où chaque étable abrite un assez grand nombre de vaches laitières.

De là, vient l'importance du choix des méthodes de contrôle qui doivent être simples et suffisamment précises pour permettre de s'assurer - sur de nombreux échantillons - de la bonne qualité bactériologique du lait et de détecter les laits anormaux (laits de mammites, laits de composition anormale, etc.).

Un problème voisin apparaît si l'on envisage le paiement du lait ou des produits laitiers suivant leur teneur en principes utiles (richesse en graisse et caséine par exemple); dans la pratique, on doit, le plus souvent, recourir à des dosages rapides, seuls utilisables pour un grand nombre d'échantillons. Aussi le perfectionnement des procédés existants, ou la mise au point de tests encore plus rapides et susceptibles par conséquent d'un emploi plus étendu, sont-ils d'un gros intérêt économique.

Il est certain en effet qu'une meilleure connaissance des variations qui se produisent dans la composition du lait d'un troupeau représente à elle seule un facteur précieux de progrès en permettant de mieux mesurer l'influence de la nutrition, du climat et des autres conditions du milieu sur la qualité du lait. Ceci offre d'autre part l'avantage d'établir les relations entre vendeur et acheteur sur des 
bases plus sûres et - les laits riches étant mieux payés que les laits pauvres - d'apporter un stimulant, sous forme d'un gain supplémentaire, à l'amélioration du bétail laitier.

\section{Les microorganismes en laiterie.}

Les procédés d'utilisation du lait font très largement appel à plusieurs groupes de microorganismes : en fromagerie ou en beurrerie, l'utilisation de souches variées de ferments lactiques et de moisissures est d'importance capitale pour assurer une bonne conservation et une maturation convenable des produits fabriqués.

Aussi le choix des souches, le maintien de leur activité en culture pure, ou simplement la réalisation des conditions de milieu les plus favorables à leur dévelopement (température, hygrométrie, aérobiose ou anaérobiose, facteurs divers de croissance ou d'inhibition) seront les principaux points à envisager. Les échecs et les accidents que l'on enregistre très souvent dans ce domaine, montrent que de sérieux progrès restent encore à faire.

\section{Les problèmes posés par la conservation et le stockage.}

La nécessité de conserver pendant plusieurs mois une partie des substances qui ne sont pas utilisables immédiatement au moment de leur production pose une troisième série de questions : celles des altérations qui surviennent au cours du stockage.

L'emploi du froid, la concentration ou la dessiccation préalables ont ouvert beaucoup de possibilités, mais de nombreux problèmes demeurent encore à résoudre.

Les altérations constatées relèvent en général de deux origines :

a) Les altérations d'origine chimique, dont un exemple bien connu est celui des défauts qui apparaissent dans le beurre renfermant une certaine quantité de cuivre;

b) Les altérations d'origine microbienne, imputables à la présence de microorganismes indésirables : ferments butyriques ou putréfiants dans les fromages, microbes thermorésistants dans les laits concentrés, moisissures et levures dans les beurres, ete.

Les pertes subies chaque année, du fait de ces altérations, sont extrêmement élevées et la mise au point de procédés susceptibles de les réduire ou même de les supprimer, représente un facteur d'amélioration considérable.

\section{La notion de qualité dans les produits laitiers.}

Les qualités organoleptiques et la "présentation " jouent, dans le cas du lait et des produits laitiers, un rôle particulièrement marqué dont dépend bien souvent la faveur du consommateur et par suite, dans une bonne mesure, la valeur économique du produit. 
A cet égard, la réputation des fromages et des beurres français - pour ne citer que deux exemples - est déjà ancienne et il est souhaitable de la conserver. Elle doit permettre en effet de tirer le meilleur parti de la variété et de l'origïnalité qui caractérisent notre production laitière, en confirmant l'existence de véritables crûs de beurre et de fromage, propres à plusieurs de nos grandes régions agricoles.

Cette notion de crû repose le plus souvent sur des observations empiriques et l'appréciation de la qualité de chacun des produits gagnera à être faite de façon plus systématique que par le passé.

Dans ce but, il serait utile de définir plus exactement la qualité (collège d'experts) et d'aborder l'étude des éléments fondamentaux sur lesquels elle repose : composition des glycérides des beurres provenant de différentes régions, propriétés de la caséine chez diverses variétés de fromage, études de la teneur en substances minérales du lait, leurs variations aveo l'origine géographique, le climat, l'alimentation, la race des vaches laitières.

\section{ORIENTATION DES RECHÊRCHÊS}

\section{Recherches relatives à la composition du lait en relation avec la physiologie de l'animal.}

Il est à peine besoin de souligner l'intérêt qu'il y a à grouper les études sur la nutrition du bétail laitier, les propriétés nutritives $\mathrm{du}$ lait et des produits laitiers, la physiologie de la lactation, enfin la génétique du bétail laitier avec celles qui portent sur le lait ou la viande proprement dits et leurs utilisations diverses.

C'est à ce niveau que s'établira une collaboration entre les recherches des zootechniciens et celles que nous poursuivons en teehnologie. Beaucoup de nos études seront d'ailleurs grandement facilitées par la possibilité de disposer d'un troupeau d'animaux d'expérience dont le Centre national de recherches zootechniques est seul à même d'assurer la constitution et l'entretien dans les meilleures conditions.

\section{Recherches de bactériologie laitière.}

Ces études doivent envisager plusieurs sujets d'aspect différent: les microbes, suivant le groupe auquelils appartiennent et suivant l'utilisation à laquelle on destine le lait, sont considérés tantôt comme des organismes indésirables que l'on s'efforce d'éliminer, de détruire ou de défavoriser le plus possible, tantôt comme des auxiliaires grâce auxquels on assure, aux moindres frais, la conservation de substances facilement altérables. Citons, à titre d'exemple, les travaux à entreprendre sur les substances bactéricides (produits 
de nettoyage, désinfectants), sur les inhibiteurs et les antibiotiques utilisés comme agents sélectifs de certaines espèces microbiennes, enfin sur les divers procédés physiques (radiations) qui contrôlent le développement des bactéries.

Parmi les microorganismes intéressants à connaître et à utiliser, on peut placer au premier rang le groupe des ferments lactiques. Ces derniers ont fait l'objet depuis une quinzaine d'années de travaux étendus tant sur le plan de la systématique que sur celui de la biochimie. Les résultats acquis sont eux-mêmes susceptibles de conduire à des applications importantes dans la pratique : nouvelles méthodes de sélection et de maintien des souches, utilisation de leur sensibilité ou de leur résistance vis-à-vis des bactériophages ou des antibiotiques, ete.

Le groupe des moisissures joue également un rôle primordial dans presque tous les procédés de transformation du lait ou des produits laitiers - ce qui justifie qu'une part leur soit réservée dans nos recherches - : phénomènes de rancissement dans les beurres, maturation de nombreux types de fromages, altérations diverses dans les laits conservés.

Les microbes producteurs de gaz (groupe du Coli-aerogenes) et les sporulés anaérobies (groupe des ferments butyriques et des ferments putréfiants) prolifèrent de façon redoutable à de nombreux stades d'utilisation du lait. L'étude des conditions qui favorisent leur développement et celle des moyens à employer pour les combattre constitue l'un des chapitres de nos travaux.

L'étude des microbes pathogènes du lait est évidemment du ressort des recherches vétérinaires et des recherches de bactériologie médicale, mais c'est également un point de rencontre où nos travaux devront être coordonnés avec ceux des spécialistes appartenant à ces disciplines, qu'il s'agisse d'étudier l'influence de divers procédés de traitement du lait sur la destruction des bactéries pathogènes (bacille de Koch, Brucella), ou du rôle que jouent les différentes infections microbiennes de la mamelle dans certaines modifications de la composition du lait.

Enfin les méthodes d'appréciation de la qualité bactériologique du lait demeurent un sujet de recherches toujours ouvert. Si certains tests ont en effet déjà rendu de grands services et permis, dans différents pays, d'améliorer notablement la qualité du lait, il reste encore beaucoup de points à préciser, notamment en ce qui concerne les conditions d'application des tests et l'interprétation des résultats. La mise au point de méthodes nouvelles et plus précises, reste également à envisager. Nous avons souligné plus haut, l'importance pratique fondamentale $\mathrm{du}$ problème de la qualité bactériologique du lait. 


\section{Recherches de chimie et physique.}

Nous ne possédons en France qu'un petit nombre de documents précis concernant l'étendue des variations de la composition du lait dans diverses régions, au cours des différentes saisons de l'année. Il serait cependant très utile que de telles analyses soient effectuées régulièrement : teneur en substances grasses et non grasses du lait, dosage des substances minérales (Ca, P), etc. Une meilleure connaissance de ces variations de composition - en dehors de son intérêt sur le plan économique et sur celui de la nutrition - aiderait à comprendre l'origine de certaines difficultés rencontrées pour l'utilisation du lait sur le plan technologique, et orienterait le travail vers la recherche des solutions les mieux adaptées au but que l'on se propose.

La place qu'occupent en France les transformations du lait en beurre et fromage conduit à réserver une large part à l'étude des phénomènes de coagulation (étude de la présure et des enzymes digestifs), aux processus de dégradation de la caséine au cours de la maturation des fromages (amino-acides et peptides libérés, etc.) aux variations dans la composition des glycérides ou des phospholipides du beurre (proportions d'acides saturés et insaturés en relation avec la texture des beurres et leurs propriétés organoleptiques). Enfin l'étude des altérations qui se produisent au cours de la conservation (oxydation, phénomènes de "vieillissement ") est également très importante.

La teneur en eau du lait ou des produits laitiers et l'affinité plus ou moins grande pour l'eau des divers constituants du lait représentent un des aspects fondamentaux qui eonditionnent la réussite de beaucoup de procédés de conservation (fromagerie, laits concentrés et laits en poudre, etc.). Aussi l'étude de l'affinité pour l'eau des diverses substances azotées, des phospholipides, etc., ne devra-t-elle pas être négligée.

Dans la pratique, les procédés de fabrication du fromage, du beurre ou du lait concentré - et aussi les moyens d'appréciation de leur qualité finale - sont basés en bonne partie sur leurs propriétés rhéologiques. L'étude systématique de ces propriétés et le remplacement d'une appréciation subjective par une mesure, au moyen d'un instrument convenable, représentent un facteur de progrès et méritent, à divers titres, de faire partie de nos recherches.

Enfin, eomme dans le cas de la bactériologie, il sera utile de procéder à de constantes mises au point et perfectionnement de nos méthodes d'analyse chimiques ou physiques. On devra se souvenir en particulier de l'intérêt que présentent les méthodes qui permet- 
tent d'effectuer, avec les mêmes ressources en personnel, et sans augmentation de frais notable, l'examen d'un plus grand nombre d'échantillons.

\title{
Conclusion
}

Les problèmes qui se posent pour la recherche laitière sont ceux que l'on rencontre dans l'étude de beaucoup de produits biologiques : si nous voulons utiliser ces derniers de la façon la plus avantageuse, - pour notre alimentation par exemple - nous devons bien connaître leur composition et leurs propriétés, savoir déterminer les limites entre lesquelles elles sont susceptibles de varier. de façon à pénétrer ensuite le mécanisme même de ces variations. Nous devons nous attacher également à l'étude et à l'amélioration de tous les procédés — dont beaucoup sont commandés par l'intervention de microorganismes - qui permettent d'utiliser de la façon la plus rationnelle le lait et les produits qui en dérivent.

Nous atteindrons ainsi le but que se propose la Recherche Agronomique dans les divers domaines auxquels elle consacre ses travaux.

\section{SUPPLEMENT TECHNIQUE}

\section{INFLUENCE DES DISPERSIONS DE CASÉINE ET AUTRES PROTÉINES SUR LA STABILITÉ AU FROID DES ÉMULSIONS DE LATEX}

\author{
par \\ G. GÉNIN \\ Ingénieur E.P.C.
}

Le latex de caoutchouc, et peut-être plus encore les latex de caoutchouc synthétique, sont aujourd'hui utilisés sur une très grande échelle dans la préparation des peintures : émulsion après addition ou non de pigments colorés. Le principal inconvénient de ces peintures émulsion est leur susceptibilité aux basses températures et par exemple, par congélation, certains de ces latex subissent une coagulation irréversible.

La coagulation des émulsions de latex sous l'influence du froid dépend de différents facteurs, comme par exemple le choix du plastifiant, l'addition de colloïdes protecteurs qui le plus souvent sont constitués par des protéines, l'addition de certains solvants et enfin le choix et la nature des pigments. 